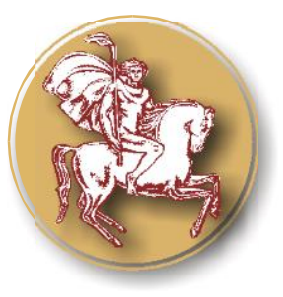

Case Report

\title{
ACUTE CORNEAL HYDROPS IN KERATOCONUS - A CASE REPORT
}

\author{
V. Haykin*, Y. Zdravkov, S. Kostova, A. Oscar, S. Dokov, Iv. Tanev \\ Clinic of Ophthalmology, University "Alexandrovska” Hospital, Sofia, Bulgaria \\ Department of Ophthalmology, Medical Faculty, Medical University - Sofia, Bulgaria
}

\begin{abstract}
Keratoconus is observed in cases of impaired corneal biochemical activity, leading to secondary thinning and ectasia. Usually the onset is in the second - third decade of life. The acute corneal hydrops occurs when intraocular aqueous passes through a rupture of Descemet membrane (DM) and is characterized with stromal edema. Estimated frequency is $2.5-3.0 \%$ among the keratoconus patient. Eye rubbing is the major risk factor. In most cases the hydrops persists for $2-4$ months. The resolution is cicatrix/scar formation, but there is a risk for complications such as vascularization, infection and corneal perforation.

We present a case of a 23 year old male patient, presenting with complains of reduced and blurry vision in the right eye and photophobia. Visual acuity and full ophthalmological examination was done. UBM was performed of the right eye. The patient received conservative therapy.

The acute corneal hydrops occurs when intraocular aqueous passes through a rupture of Descemet membrane (DM) and is characterized with stromal edema.

The treatment could be conservative or surgical. The conservative therapy includes topical lubricants, antibiotics, cycloplegics, hyperosmotic solutions, antiglaucoma drugs, topical corticosteroids and nonsteroid anti-inflammatory drugs. Surgical treatment includes intracameral injection of air or gas, but the definitive treatment is penetrating keratoplasty.

We describe a newly diagnosed patient with keratoconus, who presents with rare complication - acute corneal hydrops.

Differential diagnosis in cases of young patients with acute onset of keratitis, especially young males, should be made with acute corneal hydrops.
\end{abstract}

Key words: keratoconus, corneal hydrops, complication, cornea

\section{BACKGROUND}

Keratoconus is non-inflammatory progressive eye disorder, leading to visual impairment. (1, $2,3)$ It`s prevalence is 1 patient among 2000 individuals. $(1,3)$ It is observed in cases of impaired corneal biochemical activity, leading to secondary thinning and ectasia $(4,5)$. Usually the onset is in the second - third decade of life. The condition progresses until the fourth decade, when it stabilizes. $(6,7)$

Some studies find that the genetic background is playing an important role in the development of the disease. For example keratoconus is 4,4 times more frequent in Asians than among

\footnotetext{
*Correspondence to: Vasil Haykin, MD, 1 Georgi Sofiiski, Sofia, Bulgaria,vhaykin@gmail.com, +35929230234
}

Caucasians. (3) Some recent studies discover an inflammatory activity in keratoconus.

Interleukins (IL-1, IL6, IL8) and TNF- $\alpha$ are found in the tears of those patients. $(3,8)$ Detected are ten times greater production of PEG2 and increased respond to it. PEG2 decreases fibroblasts` activity and collagen synthesis. $(3,9)$

In the majority of cases keratoconus is an isolated disorder. However in some cases it is associated with vernal keratoconjunctivitis, atopy, Leber congenital amaurosis, Down syndrome, retinitis pigmentosa, prolapse of mitral valve and other connective tissue disorders, such as Marfan and Ehlers-Danlos syndromes. Family history is major risk factor and HLA haplotype could be used as a familial 
marker for the disease. Male gender is associated with an earlier development and more rapid progression, suggesting androgenic dependency. (3)

The acute corneal hydrops occurs when intraocular aqueous passes through a rupture of Descemet membrane (DM) and is characterized with stromal edema. $(6,7)$ The estimated frequency is $2.5-3.0 \%$ among the keratoconus patient with prevalence in young males. Unilateral involvement is more common and usually appears soon after diagnosis. Eye rubbing is the major risk factor. $(10,11)$ In most cases the hydrops persists for $2-4$ months. The resolution is scar formation, but there is a risk for complications such as vascularization, infection and corneal perforation. $(6,10)$

We present a case of hydrops as a complication of keratoconus.

\section{CASE REPORT}

HAYKIN V., et al.

We present a case of a 23 year old male patient, presenting with complains of reduced and blurry vision in the right eye, photophobia and pain for 4 days. He has been treated by general practitioner with combined topical antibiotic/corticosteroid agent, with no improvement of the symptoms. At first he was diagnosed with fungal/viral keratitis.

On the initial examination in the clinic the patient was anxious, frequently touching and rubbing his eyes. The visual acuity of right eye was 0.01 and of left eye 0.6, with normal intraocular pressure. Right eye examination showed reactive ptosis, diffuse conjunctival injection, corneal conical protrusion (Figure 1), focal area of microcystic edema and 3-4 $\mathrm{mm}$. bullae with ectasia, Munson's sign, intact corneal epithelium, with no infiltration or precipitates, deep anterior chamber, with clear fluid, photo reactive and round pupil, no abnormalities of posterior segment.

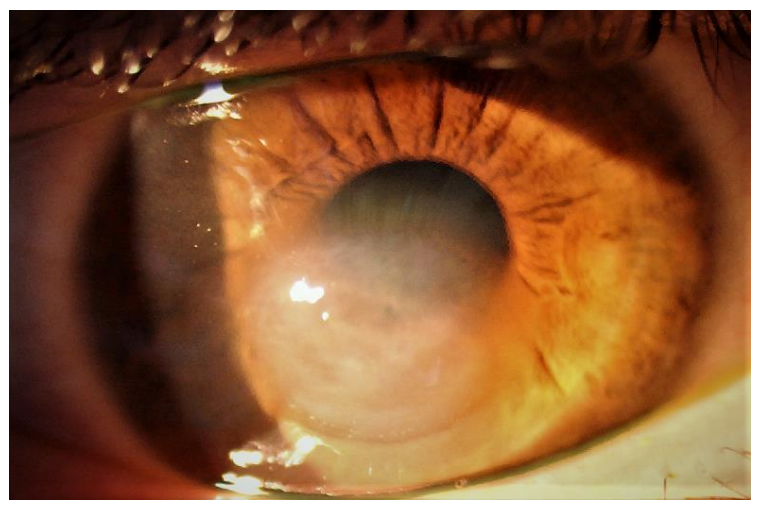

Figure 1. Right eye slit lamp examination

UBM examination of the right eye (Figure 2) showed rupture of the DM. Autokeratometry was performed to the left eye. The result was suggestive for keratoconus with high $\mathrm{K}$ readings: R1 7,52 (K1 45,25), R2 6,82 (K2 $50,60)$.

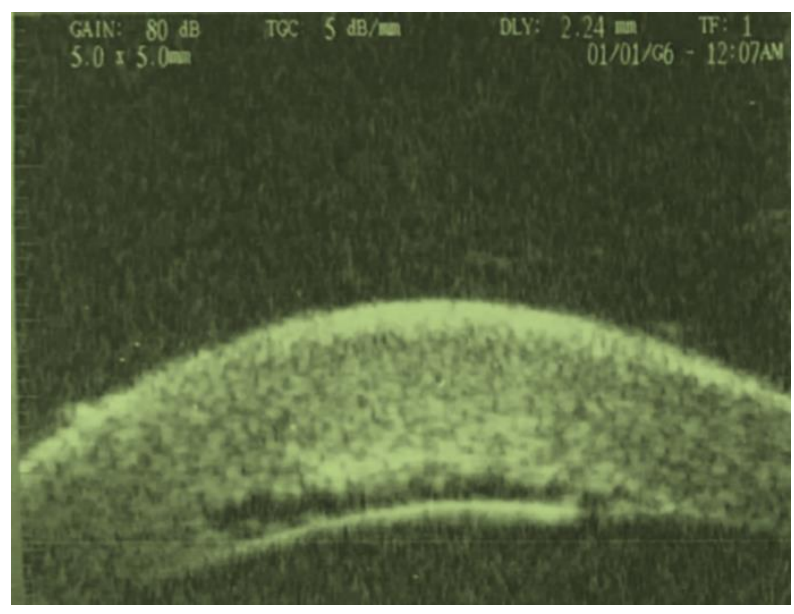

Figure 2. UBM of right eye - rupture of DM, corneal edema and filled with fluid intrastromal space. 
The patient was treated with topical and parabulbar corticosteroids, as well as topical hyperosmotic and cycloplegic therapy and was discharged on the third day with topical corticosteroids.

\section{DISCUSSION}

The acute corneal hydrops occurs when intraocular aqueous passes through a rupture of Descemet membrane (DM) and is characterized with stromal edema. $(6,7)$ Plaut (1900) first described the condition as a sudden opacification of the corneal apex caused by DM rupture. (7)

Keratoconus is observed in cases of impaired corneal biochemical activity, leading to secondary thinning and ectasia. Usually the onset is in the second - third decade of life. The condition progresses until the fourth decade, when it stabilizes. (6)

On evaluation a significantly reduced visual acuity, photophobia and pain are found. Rubbing the eyes is commonly reported. On slit lamp examination a well-defined microcystic stromal and epithelial edema, stromal cysts and injection are proved. Although the diagnosis is based on patient's history and biomicroscopy, ultrasound biomicroscopy (UBM) or optical coherent tomography of the anterior segment are recommended. (12)

The treatment could be conservative or surgical. The conservative therapy includes topical lubricants, antibiotics, cycloplegic agents, hyperosmotic solutions, antiglaucoma drugs, topical corticosteroids and non-steroid anti-inflammatory drugs. Surgical treatment includes intracameral injection of air or gas (pneumatic descemetopexy), but the definitive treatment is penetrating keratoplasty. $(6,10-12)$

Our patient's history is in line with the described cases in literature - a young male, rubbing often his eyes we can speculate that probably the progression of the undiagnosed keratoconus led to reduced visual acuity, which the patient tried to "improve" by rubbing his eyes, causing the complication.

Differential diagnosis in cases of young patients with acute onset of keratitis, especially young males, should be made with acute corneal hydrops.

\section{REFERENCES}

1. Rabinowitz YS. Keratoconus. Surv Ophthalmol;42(4):297-319, 1998.

2. Fournie P, Touboul D, Arne J-L, Colin J, Malecaze F. [Keratoconus]. $J \quad F r$ Ophtalmol;36(7):618-626, 2013. doi:10.1016/j.jfo.2013.05.004

3. Mukhtar S, Ambati BK. Pediatric keratoconus: a review of the literature. Int Ophthalmol;38(5):2257-2266, 2018. doi:10.1007/s10792-017-0699-8

4. Nikolova G. Komsiyska D. Karamalakova Y. Petkov Y. Ivanov V. Manolova T. Gadjeva V. Bulg Chem Commun, 50(C): 64-68, 2018

5. Komsiyska D. a review of the literature, TrJScie, 16(3):249-253, 2018

6. Barsam A, Petrushkin H, Brennan N, et al. Acute corneal hydrops in keratoconus: a national prospective study of incidence and management. Eye ;29(4):469-474, 2015. doi:10.1038/eye.2014.333

7. Sharma N, Mannan R, Jhanji V, et al. Ultrasound Biomicroscopy-Guided Assessment of Acute Corneal Hydrops. Ophthalmology. 118(11):2166-2171, 2011. doi:10.1016/j.ophtha.2011.03.040

8. Lema I, Duran JA, Ruiz C, Diez-Feijoo E, Acera A, Merayo J. Inflammatory response to contact lenses in patients with keratoconus compared with myopic subjects. Cornea. 27(7):758-763, 2008. doi:10.1097/ICO.0b013e31816a3591

9. Pouliquen Y, Bureau J, Mirshahi M, Mirshahi SS, Assouline M, Lorens G. [Keratoconus and inflammatory processes]. Bull Soc Belge Ophtalmol. 262:25-28, 1996.

10.Maharana PK, Sharma N, Vajpayee RB. Acute corneal hydrops in keratoconus. Indian J Ophthalmol. 61(8):461-464, 2013. doi:10.4103/0301-4738.116062

11.Grewal S, Laibson PR, Cohen EJ, Rapuano CJ. Acute hydrops in the corneal ectasias: associated factors and outcomes. Trans Am Ophthalmol Soc. 97:187-203, 1999.

12. Greenwald MF, Vislisel JM GK. Acute Corneal Hydrops.; EyeRounds.org. 2016. 